\title{
B Lymphoblastic Leukemia/Lymphoma with Hyperdiploidy
}

National Cancer Institute

\section{Source}

National Cancer Institute. B Lymphoblastic Leukemia/Lymphoma with Hyperdiploidy. NCI Thesaurus. Code C80335.

A precursor lymphoid neoplasm composed of B-lymphoblasts which contain more than 50 and usually less than 66 chromosomes. It has a favorable clinical outcome. 